\title{
L'image des armées alliées à travers les journaux des diaristes français de la guerre des Boxers
}

\section{François Pavé}

\section{(2) OpenEdition}

\section{Journals}

\section{Édition électronique}

URL : http://journals.openedition.org/abpo/223

DOI : 10.4000/abpo.223

ISBN : 978-2-7535-1512-3

ISSN : 2108-6443

Éditeur

Presses universitaires de Rennes

Édition imprimée

Date de publication : 31 décembre 2008

Pagination : 127-144

ISBN : 978-2-7535-0808-8

ISSN : 0399-0826

\section{Référence électronique}

François Pavé, «L'image des armées alliées à travers les journaux des diaristes français de la guerre des Boxers », Annales de Bretagne et des Pays de l'Ouest [En ligne], 115-4 | 2008, mis en ligne le 31 décembre 2010, consulté le 20 avril 2019. URL : http://journals.openedition.org/abpo/223 ; DOI $10.4000 / a b p o .223$ 


\title{
L'image des armées alliées à travers les journaux des diaristes français de la guerre des Boxers
}

\author{
François PAVÉ \\ Doctorant \\ CERHIO UMR 6258 - université du Maine
}

La guerre des Boxers éclate en juin 1900. La France, la Russie, l'Angleterre, les États-Unis, l'Allemagne, l'Italie, l'Autriche-Hongrie et le Japon s'unissent pour défendre leurs ressortissants présents sur le sol chinois et leurs intérêts économiques menacés par le soulèvement nationaliste des Boxers. L'ensemble de ces pays dépêche un contingent militaire qui atteint, au plus fort de la mobilisation, 107000 hommes.

Le soulèvement des Boxers se déroule pour l'essentiel dans le nord-est de la Chine, dans la province du Zhili où se situent les villes de Pékin et Tien-Tsin. Le mot Boxer vient de l'anglais ${ }^{1}$. C'est le nom que l'on donne en 1900 aux insurgés qui se réclament de la société secrète des Yihetuan ${ }^{2}$, milices de justice et de paix en chinois. Les adeptes de cette société secrète pratiquent une sorte de boxe sacrée. On trouve là l'origine du nom que leur donnent alors les Occidentaux. Les Boxers sont profondément hostiles à la présence étrangère sur le sol chinois. Cette dernière est relativement récente : à la suite de la première guerre de l'opium, qui oppose les Britanniques à la Chine de 1839 à 1842, les Anglais, par le traité de Nankin, obtiennent le droit de commercer dans l'Empire du Milieu jusqu'alors replié fermement sur lui-même. L'ensemble des nations occidentales et le Japon obtiennent, par la suite, des droits identiques. En 1895, la défaite chinoise face à l'Empire du Soleil-Levant accélère le phénomène. La faiblesse de la

1. On trouve dans les sources le mot anglais Boxer et le mot français Boxeur. Les spécialistes français actuels de ce sujet utilisent le mot anglais. J'ai donc choisi de faire de même dans les pages qui suivent.

2. Les membres de cette société secrète pratiquaient les arts martiaux, c'est pourquoi les Britanniques leur donnèrent le nom de Boxers. Voir DunstheIMER, Guillaume, "Le mouvement des Boxeurs ", Revue Historique, n 231, avril-juin 1964, p. 387-416 et HoANG, Michel, "L'été chaud de Pékin de 1900 : Le siège de Pékin ", L'Histoire, mai 1990, n 133, p. 76-79. 
Chine, qui tarde à se moderniser, apparaît alors au grand jour et l'Empire du Milieu devient un eldorado où les étrangers se pressent pour faire des affaires.

\section{Les origines du conflit}

La présence étrangère est vécue par la plupart des Chinois comme une véritable humiliation. Les Célestes sont en effet habités par un fort sentiment national. Jusqu'alors convaincus de la supériorité de leur culture face à celle des " Barbares ${ }^{3}$ ", les Chinois éprouvent un profond ressentiment à l'égard des Occidentaux en particulier depuis l'adoption d'une loi d'exclusion frappant les Chinois aux États-Unis en 1882 et l'imposition d'une forte taxe à l'entrée des migrants chinois au Canada en 1885. Si les Occidentaux ne tolèrent pas leur présence sur leur sol, il paraît légitime aux Chinois de ne pas accepter celle des Occidentaux sur le leur. C'est donc dans ce contexte qu'éclate le soulèvement des Boxers.

Les premiers troubles se développent dans la province du Shandong dès 1898. Au début du printemps 1900, ils touchent principalement le Zhili. La situation se dégrade en juin. Les Boxers détruisent les voies ferrées, les lignes télégraphiques et menacent les étrangers. Il y a des massacres de missionnaires et de civils. Le 13 juin les Boxers entrent dans Pékin. Répondant à des provocations des Occidentaux, ils incendient des églises et massacrent des chrétiens. Quelques jours plus tard, ils se rendent maîtres de Tien-Tsin, grande ville située entre Pékin et la mer. Inquiètes, les puissances occidentales et japonaise décident d'unir leurs forces dans une grande coalition internationale et dépêchent des troupes pour rétablir le calme.

Sentant la menace se préciser, les Occidentaux avaient envoyé, dès le début du mois de juin, un premier contingent vers les côtes chinoises. Les premiers navires arrivent dans la baie de Takou dès le 4 juin. Le 17 juin Occidentaux et Japonais s'emparent des forts marins de Takou, port situé dans le golfe de Pétchili, et commencent à progresser sur le sol chinois. Cette attaque brutale scandalise l'impératrice $\mathrm{Ci}-\mathrm{X}$. Elle prend la décision de soutenir le mouvement boxer et déclare la guerre aux puissances étrangères le 20 juin. Le même jour, les opinions publiques internationales mesurent la gravité de la situation en apprenant l'assassinant, dans une rue de Pékin, du ministre d'Allemagne en Chine ${ }^{4}$, le baron Von Ketteler, qui se rendait au Tsong-Li-Yamen, le ministère des Affaires étrangères chinois. On peut s'interroger sur le fait que le quartier des légations de Pékin parvienne à résister à un siège de 55 jours. L'explication se trouve dans le fait que le commandant chinois des forces armées du nord, n'étant pas en accord avec la décision de l'impératrice d'engager un conflit contre les étrangers, refuse

3. Pour les Chinois, les barbares sont alors tous les peuples qui ne parlent pas le chinois.

4. C'est le titre que l'on donne alors aux ambassadeurs. 
aux assiégeants l'utilisation du matériel moderne dont dispose en réalité l'armée chinoise ${ }^{5}$. Le 14 juillet, les Occidentaux repliés dans le quartier des légations de Tien-Tsin sont libérés par des soldats de la force internationale. Le 15 août c'est le tour des assiégés de Pékin.

Dès lors, les combats cessent progressivement même si des poches de résistance demeurent. Le gouvernement chinois s'engage sur la voie des négociations de paix. Les troupes de la coalition internationale, qui ont embarqué durant l'été 1900, continuent néanmoins d'arriver en Chine pendant plusieurs semaines car le voyage en bateau depuis l'Europe dure, à l'époque, de 55 à 60 jours. Les soldats qui débarquent ont pour mission de pacifier définitivement le nord de la Chine. Cette présence militaire permet aussi de faire pression sur les autorités chinoises pour obtenir des conditions avantageuses lors des pourparlers de paix. Le nombre de soldats engagés par chaque nation dans le conflit reflète les prétentions de chacune en Chine. Le contingent étranger reste présent dans l'Empire du Milieu de façon massive jusqu'au mois de mai 1901.

Nombre de soldats engagés par chaque pays durant la campagne de Chine (1900-1901) ${ }^{6}$

\begin{tabular}{|l|c|}
\hline \multicolumn{1}{|c|}{ Pays } & Nombre de soldats \\
\hline France & 17000 \\
Allemagne & 22500 \\
Angleterre & 20000 \\
Russie & 18000 \\
Japon & 22000 \\
États-Unis & 5100 \\
Italie & 2200 \\
Autriche & 500 \\
\hline Total général & 107300 \\
\hline
\end{tabular}

Les soldats français, au nombre de 17000 , sont pour la plupart volontaires pour participer à la campagne de Chine. Certains d'entre eux, conscients qu'ils vivent des moments exceptionnels, procèdent à la rédaction de journaux de campagne. Ils y consignent des informations diverses, des observations personnelles, des émotions ressenties. Ces journaux constituent une source originale pour l'historien, dans la mesure où il s'agit du regard porté sur les événements de Chine par de simples soldats. Le contenu de ces journaux est par conséquent bien différent de celui des rapports officiels. À ce jour, trois journaux de soldats sont connus.

5. BASTID, Marianne, Bergère, Marie-Claire et ChesneAuX, Jean, Histoire de la Chine, l'illusoire modernité, 1885-1921, Paris, Hatier Université, 1972, p. 80

6. Bourgerie, Raymond et Lesouef, Pierre, La Guerre des Boxers, Tseu-Hi évite le pire, Paris, Éditions Economica, 1998, p. 127. 


\section{Trois journaux de soldats}

Le journal de Léon Silbermann a été publié en $1910^{7}$. Léon Silbermann est un soldat à la carrière modeste puisque, après 15 années au service de l'infanterie de marine, il ne brigue que le grade de caporal. Il apparaît comme l'archétype du soldat honnête, droit et courageux. Son discours est toujours " militairement correct ". Fantassin, il participe, avec ses camarades, à de nombreuses colonnes à travers la campagne du Zhili. Il est très souvent en contact avec les soldats des armées alliées. Il nous fait part, à de nombreuses reprises, de ses impressions relatives à ce sujet.

Le journal de Jules Bedeau a été publié, dans son intégralité, en 1992 dans la revue d'histoire locale mayennaise $L^{\prime} O$ ribus $^{8}$. Le texte y est proposé brut, sans explication. En 1900, Jules Bedeau est un jeune soldat originaire de la Mayenne qui, pour mettre fin à la monotonie d'un service militaire qui dure alors 3 ans et qu'il effectue dans l'artillerie au Mans, décide de se porter volontaire pour participer à la campagne de Chine. Il souhaite voir du pays et " vivre le baptême du feu ${ }^{9}$ ". Il arrive en Chine le 14 octobre alors qu'un protocole rétablissant la paix est signé avec les autorités chinoises depuis le 7 septembre. Néanmoins, il passe neuf mois en Chine avec son régiment dont la mission est d'assurer la surveillance du centre du Zhili. Jules Bedeau ne participe à aucun combat mais travaille à la reconstruction de la province détruite.

Victor Petit est le troisième diariste connu de la guerre des Boxers. Son journal a été publié en 1996; il est accompagné d'un commentaire réalisé par l'historien Alain Dalotel ${ }^{10}$. En 1900, Victor Petit est un tout jeune soldat qui vient de s'engager dans l'infanterie et qui se porte volontaire pour participer à la campagne de Chine. Son unité est une des toutes premières à arriver sur le théâtre d'opérations car elle est, avant les événements, cantonnée en Indochine. Les troupes françaises en Chine, fraîchement arrivées, ne sont pas encore bien organisées sur le plan logistique. De ce fait, les soldats souffrent de la faim et du froid. Certains acceptent avec difficulté la discipline nécessaire au bon déroulement d'une campagne militaire. C'est le cas de Victor Petit qui déserte à deux reprises. La seconde fois il se cache durant deux mois parmi la population chinoise. Il en profite pour s'initier au chinois et découvrir la culture chinoise pour laquelle il ressent respect et admiration. Retrouvé par l'armée française, il est condamné à dix ans de bagne en Guyane. On perd ensuite sa trace. Lors du conflit, il envoie son journal de façon régulière à sa famille. Alain Dalotel a ajouté au journal de

7. SilBermann, Léon, Souvenirs de campagne, Paris, Plon-Nourrit, 1910, 281 p.

8. Le journal de Jules BEDEAU est l'objet d'une nouvelle publication. Le journal est précédé d'un long commentaire qui repose sur un travail comparatif avec les autres journaux évoqués dans cet article : PAVÉ, François, Le Journal de Jules Bedeau, Un soldat français dans la Chine des Boxers, Paris, Éditions You-Feng, 2007, 227 p.

9. Jules Bedeau, le 27 octobre 1900, dans Pavé François, Le journal de Jules Bedeau, op. cit., p. 145.

10. DALOTEL, Alain, De la Chine à la Guyane. Mémoires du bagnard Victor Petit 1879-1919, Paris, La boutique de l'Histoire, 1996, 325 p. 
Victor Petit des lettres que ce dernier avait adressées à sa famille durant la campagne de Chine.

Aux témoignages de ces trois soldats, on peut ajouter le journal rédigé par l'officier Pierre Loti ${ }^{11}$. Pierre Loti, né Louis Marie Julien Viaud, ancien officier de marine et écrivain français, élu à l'Académie française en 1891, est l'auteur de romans inspirés par ses voyages. Son œuvre est marquée par le goût pour l'exotisme. En 1900, il est âgé de cinquante ans. Passionné par l'Extrême Orient, il décide de se porter volontaire pour participer à la campagne de Chine. Il est alors capitaine de vaisseau. Il arrive au large de la Chine, le 24 septembre 1900, à bord du Redoutable, le navire amiral de la flotte française dirigée par l'amiral Pottier. Comme Jules Bedeau, Pierre Loti arrive dans une Chine écrasée par l'occupation. Son parcours en Chine ressemble davantage à une promenade touristique qu'à une expédition militaire.

Ce n'est pas le cas de Georges Weulersse ${ }^{12}$, jeune historien qui voyage en Extrême-Orient grâce à une bourse de la fondation Albert Kahn attribuée par l'université de Paris ${ }^{13}$ et qui arrive dans le golfe du Pétchili le 18 juin alors que les combats font rage. Les forces européennes ont attaqué, la veille, les forts chinois de Takou sensés protéger la côte de tout débarquement. Des corps éventrés jonchent le sol. Les ruines fument encore. Georges Weulersse rapporte ses observations dans un journal qu'il rédige du 13 juin au 4 août. Ce journal fut publié dans la revue Le Tour du monde : nouveau journal des voyages du second semestre 1901.

Les journaux de Léon Silbermann, Jules Bedeau et Victor Petit, dont la lecture est enrichie par ceux de Pierre Loti et Georges Weulersse, constituent un corpus cohérent dans la mesure où ces soldats ont été confrontés au même contexte, au même moment, dans la même province chinoise. La similitude des situations rend intéressant un travail comparatif de leurs observations sur les différentes armées engagées dans le conflit. Car c'est bien son caractère international qui caractérise ce conflit. Le regard que ces diaristes portent sur les soldats allemands qui font partie des forces européennes retient tout particulièrement l'attention trente ans après la guerre de 1870 et quatorze ans avant la Grande Guerre. Il apparaît aussi essentiel d'étudier l'évolution de ce regard au cours de la campagne.

\section{Le début de la campagne et la nécessaire entente entre les soldats des armées de la coalition internationale}

C'est en arrivant dans la baie de Takou que Jules Bedeau constate l'importance de la coalition réunie pour l'expédition de Chine. Toutes les

11. LOTı, Pierre, Les derniers jours de Pékin, Paris, Éditions Balland, 1985, 275 p.

12. Weulersse, Georges, Au Petchili, et sur les frontières de Mandchourie, Paris, 1900, 36 p. Georges Weulersse est un historien qui est alors en voyage dans cette partie du monde. Il arrive en baie de Takou sur un navire de guerre.

13. Georges Weulersse sera par la suite professeur agrégé d'histoire à Orléans. En 1910, il soutient une thèse sur les physiocrates. Il a enseigné à l'ENS de Saint-Cloud. 
nations engagées sont présentes : «Plus nous approchons, plus les nuages de fumée deviennent épais et nombreux : nous apercevons bientôt les navires qui les produisent. Ils sont ici un grand nombre de toutes les nations et de toutes les tailles ${ }^{14}$."

C'est aussi le premier contact que Georges Weulersse a avec cette coalition $^{15}$. L'importance du regroupement est pour lui, comme pour Jules Bedeau, source d'admiration. Le caractère considérable de l'escadre semble rendre concrète aux yeux de ces deux diaristes la détermination des nations engagées dans l'expédition de Chine. Le 18 juin, Georges Weulersse estime à trente-cinq le nombre de navires étrangers présents. Le 17 octobre, Jules Bedeau compte quatre-vingt-six à quatre-vingt-sept bateaux. Ainsi, en l'espace de quatre mois les renforts sont-ils arrivés. Jules Bedeau rapporte qu'il existe une réelle coordination entre les différentes nations. Celles-ci respectent en effet des règles communes : "Sitôt après le coucher du soleil, nous entendons plusieurs coups de canon et les couleurs sont descendues de tous les navires aussi bien étrangers que français ${ }^{16}$."

Jules Bedeau évoque ensuite la présence des troupes occidentales et japonaises dans les forts de Takou ${ }^{17}$ qui ont été pris le 17 juin ${ }^{18}$. Sur ces forts, il observe la présence des pavillons russes, italiens et anglais. Il ne fait pas de remarque sur l'absence du drapeau français alors que Georges Weulersse, lui, semble s'en inquiéter : "Nous repassons devant les forts; on distingue bien cette fois tous les drapeaux : ceux de l'Italie, d'Angleterre et du Japon sur la rive gauche; ceux de Russie et d'Allemagne sur la rive droite. Le nôtre n'y est pas encore ${ }^{19}$."

L'honneur de l'armée française semble ici, en quelque sorte, menacé, mais Georges Weulersse, comme pour rassurer le lecteur, rappelle quelques lignes plus loin, que la France a joué un rôle déterminant dans la prise de ces forts alors que la bataille semblait mal engagée ${ }^{20}$. La canonnière française, le Lion, a en effet réussi à envoyer un obus sur un des forts où étaient stockées les munitions chinoises. Cela a provoqué une énorme explosion dans un des forts. Cette action fut décisive et permit aux soldats alliés de pénétrer dans les fortifications. Jules Bedeau rapporte aussi cette anecdote qui lui a été racontée par des matelots. Il réserve un long paragraphe à la description de cet événement qui lui apparaît important pour l'image de l'armée française ${ }^{21}$. Le général Voyron, dans son Rapport sur l'expédition de Chine, y fait aussi allusion, mais il affirme que le Lion " a pris une part brillante à cette action, au cours de laquelle plusieurs bâtiments des flot-

14. BEDEAU, Jules, Journal..., op. cit., p. 12.

15. Weulersse, Georges, Au Petchili..., op. cit., p. 98.

16. BEDEAU, Jules, Journal..., op. cit., p. 13.

17. Ibidem, p. 15.

18. WeulersSE, Georges, Au Petchili..., op. cit., p. 98.

19. Ibidem, p. 104

20. Ibid., p. 100.

21. BEDEAU, Jules, Journal..., op. cit., p. 15. 
tes alliées avaient subi de fortes avaries ${ }^{22}$ ". Il ne parle pas de rôle décisif comme l'ont fait les deux auteurs précédents. Ainsi, si nos diaristes se réjouissent du caractère international de la coalition, il leur paraît important de préciser à leurs futurs lecteurs le rôle essentiel que joue l'armée française au sein de celle-ci.

Le jour même de son arrivée sur le sol chinois, Jules Bedeau constate la présence des nombreuses troupes étrangères. Dès sa descente du bateau, il traverse des cantonnements japonais, allemands et russes. Il écrit : "Pour cela nous traversons les cantonnements japonais, allemands et russes. Eux sont bien logés, ils sont dans un quartier qui n'a presque pas souffert de la guerre. Mais quand nous approchons du côté où nous devons passer la nuit, ce ne sont plus que ruines ${ }^{23}$."

Il semble y avoir dans les propos de Jules Bedeau une certaine jalousie vis à vis de la situation matérielle des soldats des autres pays. Victor Petit constate aussi à plusieurs reprises que les conditions matérielles des soldats français sont moins bonnes que celles des autres nations : "C'est avec des ânes boiteux, pelés, tondus, harnachés à la diable que plus tard nous fîmes notre entrée à Pékin, alors que les autres nations, particulièrement Allemands et Anglais, possédaient en abondance de superbes mulets équipés tout à neuf avec des harnais en cuir fauve, rutilants ${ }^{24}$."

À plusieurs reprises Victor Petit critique le manque de moyens et d'organisation de l'armée française. On peut peut-être avancer comme explication le fait que la France, avant le soulèvement des Boxers, était très peu présente dans la région du Zhili. La zone de présence française en Chine, en 1900, se situe au sud-ouest de ce vaste pays, dans le Yunnan, et non au nord-est comme c'est le cas pour la Russie et l'Allemagne. La situation des soldats japonais est aussi plus favorable du fait de la proximité géographique avec la Chine. Léon Silbermann fait aussi à plusieurs reprises ce type de remarques sur le mauvais équipement et la mauvaise organisation de l'armée française ${ }^{25}$. Vraisemblablement conscient de cette situation, l'étatmajor français demande à l'ensemble de ses militaires présents en Chine d'observer les pratiques des autres armées. Il s'agit d'opérer une sorte d'espionnage afin d'améliorer le fonctionnement de l'armée française :

«Un jour, par la voie de l'ordre, le général en chef prescrivit dans toutes les armes, de demander aux militaires de tous grades leur avis sur tout ce qu'ils avaient pu remarquer d'intéressant dans les troupes des autres nations et d'indiquer les moyens susceptibles d'alléger ou de modifier le service en campagne ${ }^{26}$."

22. Voyron, Général Émile, Rapport sur l'expédition de Chine (1900-1901), Paris, Charles Lavauzelle, 1904, 514 p.

23. BEDEAU, Jules, Journal..., op. cit., p. 17.

24. DALOTEL, Alain, De la Chine à la Guyane..., op. cit., p. 97.

25. SilBermann, Léon, Souvenirs..., op. cit., p. 195, 209, 268.

26. Ibidem, p. 239 
Si la guerre des Boxers constitue pour les militaires français le moment idéal pour observer les pratiques des armées de la coalition, c'est aussi l'occasion de montrer leur maîtrise de l'art militaire. C'est ce que souligne le général Lelong dans le discours qu'il prononce, dans la cour de la gare du Mans, lors du départ de Jules Bedeau et de ses trente et un compagnons :

«L'Europe entière aura les yeux sur vous. Vous représenterez notre artillerie devant les armées des premières puissances du monde et vous devez faire voir la valeur de notre canon. Je vous félicite, mes amis, de votre prompte détermination et même je vous envie. Le monde entier vous suivra dans votre campagne et vos combats ${ }^{27}$."

Même si elles s'observent mutuellement, la collaboration entre les armées des forces étrangères apparaît réelle à la lecture des différents témoignages. Les soldats des armées coalisées circulent dans les mêmes bateaux et dans les mêmes trains. Les Russes assurent la protection de la voie ferrée qui va de Takou à Pékin et qui est empruntée par l'ensemble des forces internationales. Ils saluent et font des signes d'amitié à Jules Bedeau et ses camarades quand ils passent devant leurs postes de garde ${ }^{28}$. En gare de Yang-Tsoun toutes les nations se côtoient et coopèrent. Le train dans lequel dort Jules Bedeau durant la nuit du 27 au 28 octobre 1900 est occupé par des Allemands, des Russes et des Japonais. Les sentinelles allemandes assurent la protection de tous ${ }^{29}$.

Léon Silbermann confirme à de nombreuses reprises la bonne entente entre l'ensemble des nations présentes. Les relations entre les différentes armées occupent une part importante de son récit. Les soldats coopèrent, se soutiennent, participent à des missions communes ${ }^{30}$. Léon Silbermann donne une explication à cette entente parfaite entre les troupes des différentes nations : «En Chine, et surtout au début des opérations, ce furent les privations et les dangers que nous partagions qui rendirent cette camaraderie internationale si charmante et si utile ${ }^{31}$."

Ainsi, aux yeux du soldat Silbermann, est-ce avant tout par nécessité que les soldats coopèrent. Cette situation permet aux armées de se découvrir et même parfois de s'apprécier. Cette proximité se traduit par des scènes assez surréalistes quatorze ans avant la première guerre mondiale : les soldats discutent au sujet de leurs armes respectives. Jules Bedeau nous apprend qu’à Tien-Tsin, des officiers russes et allemands viennent observer le matériel de sa batterie et que « certains même viennent nous demander des renseignements sur nos pièces ${ }^{32}$ ". Jules Bedeau compare la conception de son fusil avec celui d'Américains, de Russes et d'Allemands lors d'un

27. Discours rapporté dans le journal La Sarthe, le 9 août 1900.

28. BEDEAU, Jules, Journal..., op. cit., p. 18.

29. Ibidem, p. 19.

30. SilBermann, Léon, Souvenirs..., op. cit., p. 188.

31. Ibidem, p. 190.

32. BEDEAU, Jules, Journal..., op. cit., p. 20. 
transfert en train qui le mène vers Tonkou. Georges Weulersse ${ }^{33}$ et Léon Silbermann ${ }^{34}$ observent le même type de scène.

Il faut préciser que si cette collaboration entre les soldats des différentes nations est intense sur le terrain, elle l'est aussi à l'échelon le plus élevé. Les gouvernements échangent en permanence par télégrammes ou par courrier et les décisions importantes sont prises en commun accord en plus haut lieu ${ }^{35}$.

Sur le terrain, la solidarité entre les nations ne revêt pas uniquement un caractère positif. Si les alliés sont unis pour le meilleur, ils le sont aussi parfois pour le pire. Les soldats de toutes les nations se livrent à des pillages systématiques ${ }^{36}$. Victor Petit le constate alors qu'il occupe la fonction de "policier " dans Pékin pacifié ${ }^{37}$. Il assure en théorie la sécurité des habitants et prévient le pillage des maisons abandonnées ou en partie détruites mais dans les faits, il pratique les mêmes exactions que les autres soldats. Il écrit d'ailleurs à son frère, sans craindre de le choquer, " inutile de te dire que je pille plus que tous les autres. » et il précise : «[...] si je m’aperçois qu'une maison est inhabitée, j'y fait une perquisition à hauteur, j'ai une splendide couverture pour me couvrir, des coussins de soie comme matelas, un manteau en fourrure qui vaudrait en France une centaine de francs, une trentaine de mètres de soie surali, de l'encre de Chine et des petites chinoiseries ${ }^{38}$."

L'américain, Jasper Whiting, correspondant de guerre pour la Westminster Gazette de Londres, confirme le fait que le pillage est largement pratiqué en Chine. Les soldats, les officiers et les civils européens vident littéralement les maisons et les magasins désertés par les Chinois. Les membres de toutes les nations étrangères présentes y participent sans exception ${ }^{39}$. La question du pillage éclabousse l'ensemble des armées alliées, même si la presse de chaque pays affirme que ces agissements sont uniquement le fait des soldats des autres pays de la coalition.

Léon Silbermann, animé par un sentiment de solidarité pour les soldats de l'ensemble des nations, conteste ces propos et affirme que la presse, qui dénonce ces agissements dans chaque pays en les attribuant aux soldats des autres nations, s'est montrée injuste et il apporte une explication aux pillages :

\footnotetext{
33. WeulersSe, Georges, Au Petchili..., op. cit., p. 100.

34. Silbermann, Léon, Souvenirs..., op. cit., p. 270.

35. Ministère des Affaires Étrangères, Documents diplomatiques, Chine 1899-1900, Paris, 1901.

36. Petrt, Victor, dans : Dalotel, Alain, De la Chine à la Guyanne. Mémoires du bagnard Victor Petit 1879-1919, Paris, La boutique de l'Histoire, 1996, p. 107. Voir aussi un article de L'Illustration rédigé par Stéphane LAUZANE le 12 janvier 1901 qui met aussi en doute l'attitude de l'ensemble des armées engagées dans le conflit.

37. DAlotel, Alain, De la Chine à la Guyane, Mémoires du bagnard Victor Petit 1879-1919, Paris, La boutique de l'histoire éditions, 1996, p. 296.

38. Ibidem, p. 296.

39. Whiting, Jasper, China, 1900 : The Eyewitnesses Speak, Londres, Green-Hill Books, 2000 , p. 222 et p. 223.
} 
" Il est entièrement faux que le pillage de Pékin soit le fait des soldats européens. La plus grande partie avait été mise à sac, avant l'entrée des troupes alliées, par les Boxers et les soldats de Tong Fou-Sian qui s'y étaient livrés à tous les excès pendant deux mois et avaient brûlé des quartiers entiers. Les Européens y sont entrés affamés et manquant de tout; il est très naturel qu'ils soient allés chercher des vivres dans des maisons non habitées. [...] Une police très rigoureuse, sous la direction du commandant Brenot, fut organisée pour empêcher le pillage et permettre la reprise du commerce $^{40}$."

Si les propos de Léon Silbermann apparaissent en partie fondés (les Boxers avaient procédé à un premier pillage de Pékin avant l'arrivée des Occidentaux, comme s'accordent à le reconnaître les historiens aujourd'hui), il est indéniable que les alliés se sont appliqués à poursuivre l'œuvre entamée. Ainsi les alliés semblent-ils s'entendre aussi bien pour le meilleur que pour le pire.

\section{Le cas particulier des relations franco-allemandes}

Trente ans après la guerre de 1870, il apparaît intéressant d'observer quel regard portent les diaristes français, souvent éduqués à l'école communale dans l'esprit de revanche, sur les soldats allemands aux côtés desquels ils combattent et vivent en Chine. Dans le manuscrit de Jules Bedeau, aucune remarque ne témoigne d'une quelconque animosité, d'un quelconque esprit de revanche à l'égard des Allemands. Pour le soldat Bedeau, les soldats de Guillaume II apparaissent comme des alliés au même titre que les soldats des autres nations. Jules Bedeau, qui sait que son journal sera lu par ses proches, n'hésite pas à donner une image positive des Allemands. Il ne s'exprimerait sans doute pas ainsi si les relations entre Français et Allemands ne s'étaient pas considérablement améliorées.

En effet, au tournant du siècle, des signes comme la présence de navires français à l'inauguration du canal de Kiel en 1895 ou la présence allemande à l'exposition universelle de Paris de 1900, témoignent d'une certaine détente, voire d'un rapprochement entre les deux pays. Ce rapprochement est d'autant plus facile que, vue d'Allemagne, la France est devenue une nation moyenne et qu'elle ne constitue plus une réelle menace ${ }^{41}$.

Léon Silbermann décrit aussi à de nombreuses reprises la bonne entente qui caractérise les relations entre soldats français et allemands. Parlant luimême la langue de Goethe, il communique facilement avec eux et il observe à maintes reprises la volonté des hommes de Guillaume II de se rapprocher de lui et de ses compagnons : "J'ai remarqué que les soldats allemands surtout cherchaient à se rapprocher de nous et à nous être agréables de

40. Silbermann, Léon, Souvenirs..., op. cit., p. 199.

41. KRUMHEIch, Gerd, " La puissance militaire française vue d'Allemagne autour de 1900 ", La puissance française à la belle époque, p. 199-209. 
toutes façons ${ }^{42}$. " " [...] Je suis obligé de leur rendre cette justice que pendant cette pénible campagne, les allemands ont souvent saisi les occasions de nous être agréables ${ }^{43}$."

L'entente entre soldats des deux nations sera encore améliorée lorsque des soldats français parviendront à sauver le maréchal Von Waldersee pris au piège dans sa résidence en feu ${ }^{44}$. Pierre Loti précise alors : « Dans l'aprèsmidi [le 20 avril 1901], le maréchal Von Waldersee vient au quartier général français. Il se complaît à redire, ce qui est du reste la vérité, que l'incendie a été éteint presque uniquement par nos soldats, sous la conduite de mon nouvel ami, le colonel Marchand ${ }^{45}$."

Léon Silbermann indique d'ailleurs qu'après cet épisode fâcheux, les soldats français reçoivent de l'empereur Guillaume II une lettre de remerciement pour leur bravoure ${ }^{46}$. Ces bonnes relations franco-allemandes s'observent aussi au niveau des états-majors. C'est ce que l'on peut constater à la lecture du Rapport sur l'expédition de Chine du général Voyron. Le général en chef des forces françaises y décrit la très bonne qualité de ses relations avec le général Von Waldersee. Les points de vues des deux hommes sont les mêmes et les décisions sont prises d'un commun accord.

Précisons que, très tôt, Guillaume II entrevoit la nécessité d'un rapprochement entre les nations européennes pour qu'elles puissent s'imposer durablement en Chine. La première raison nécessitant un rapprochement est la protection des chrétiens menacés dans l'Empire du Milieu. Ainsi, dans Le Monde illustré du 30 novembre 1895, peut-on voir une allégorie commandée par Guillaume II à un peintre allemand, représentant les nations européennes sous la forme de femmes unies derrière saint Michel, armé d'une épée, pour aller protéger les chrétiens de Chine menacés ${ }^{47}$. Cette préoccupation précoce quant à la situation des Européens en Chine explique pourquoi l'Allemagne se voit attribuer le haut commandement des opérations. Le choix du général Von Waldersee pour diriger l'ensemble des troupes alliées constitue aussi une marque de rapprochement entre la France et l'Allemagne. Cette décision est le fruit de négociations menées avec beaucoup de sérénité par l'ensemble des dirigeants des nations présentes dans l'Empire du Milieu ${ }^{48}$. La raison principale qui motive cette décision est le fait que l'Allemagne a été la plus touchée, sur le plan symbolique, par le soulèvement des Boxers du fait de l'assassinat, dans une rue de Pékin, de son ministre en Chine, le baron Von Ketteler, le 20 juin 1900 par un soldat de l'armée régulière chinoise. Il faut préciser que ce choix n'est pas partagé par tous. Une partie de la presse n'est pas satisfaite par cette décision.

42. Silbermann, Léon, Souvenirs..., op. cit., p. 189.

43. Ibidem, p. 201.

44. Ibid., p. 248.

45. Lot, Pierre, Les derniers jours..., op. cit., p. 203.

46. Silbermann, Léon, Souvenirs..., op. cit., p. 249.

47. Le Monde illustré, le 30 novembre 1895, p. 354.

48. Cf. Ministère des Affaires Étrangères, op. cit., Paris, 1901. 
Ainsi, le journal La Sarthe proteste-t-il contre la nomination du général Von Waldersee : "Mais qu'il [le général Von Waldersee] donne des ordres à un soldat français parce qu'il est maréchal allemand, jamais ${ }^{49}$ ! "

Cette hostilité vis-à-vis de l'Allemagne se manifeste dans certains journaux. Ainsi, dans la presse nationaliste, reproche-t-on à l'Allemagne d'avoir armé et entraîné l'armée chinoise qui combat aux côtés des Boxers : "Si bien que le soulèvement formidable des fanatiques chinois contre les étrangers est en ce moment servi - et trop efficacement, hélas! - par les leçons que l'empereur Guillaume a prodiguées à ses élèves. [...] Non! Il ne devrait plus être colonel russe, ni colonel anglais, ni colonel autrichien - ou bien alors il devrait être également général chinois : Boxer en chef ${ }^{50}$ ! " Ces propos contrastent avec les bonnes relations décrites par nos diaristes de la guerre des Boxers. Charles Laurent a raison quand il affirme que l'Allemagne avait armé et formé les troupes chinoises, mais elle n'était pas la seule puissance européenne dans cette situation. La France avait aussi participé à la formation et à l'entraînement de l'armée chinoise.

Ce sentiment d'hostilité à l'égard des Allemands observé dans la presse nationaliste s'explique aussi par le problème de la perte de l'Alsace-Lorraine qui demeure, pour certains, un obstacle à tout rapprochement. Dans les écrits des diaristes de la campagne de Chine, il n'est jamais fait mention de ce problème spécifique des relations franco-allemandes. Il est vrai que cet attachement aux régions perdues est, en 1900, moins fort qu'auparavant dans la population française ${ }^{51}$. Les soldats Bedeau, Silbermann et Petit sont nés après la guerre de 1870. Cela explique peut-être pourquoi ils semblent assez peu sensibles à ce problème. Peut-être l'étaient-ils quand même un peu, mais pour nos trois diaristes la situation de l'Alsace-Lorraine n'est pas un sujet qui s'impose quand ils évoquent leurs relations avec les soldats allemands.

Si l'image des soldats de Guillaume II donnée par nos trois diaristes est positive, il faut néanmoins souligner que ces derniers s'autorisent néanmoins quelques critiques. Car si les soldats allemands se montrent agréables à l'égard des soldats français, Léon Silbermann évoque à plusieurs reprises l'extrême violence de leur attitude à l'égard de la population chinoise. Il affirme qu'ils n'hésitent pas à voler, à frapper et à tuer les Chinois ${ }^{52}$ : "Partout où les Allemands passaient, ils bombardaient, brûlaient et tuaient tout sur leur passage ${ }^{53}$." Les propos guerriers que tient Guillaume II à Bremerhaven, le 27 juin 1900, devant ses troupes qui embarquent pour l'Empire du Milieu expliquent peut-être, en partie, cette attitude extrême. Il les invite à venger l'assassinat du baron Von Ketteler perpétré sept jours plus tôt :

49. La Sarthe, le 10 août 1900.

50. LAURENT, Charles, L'Éclair, le 10 juillet 1900.

51. PoIDEVIN, Raymond et BARIÉTY, Jacques, Les Relations franco-allemandes, 1875-1975, p. 150 à 153 .

52. Ibidem, p. 224, 228, 240, 243 et 248.

53. SilbermanN, Léon, Souvenirs..., op. cit., p. 240. 
" Soyez des exemples de force virile et de discipline, de dépassement et de maîtrise de soi. L'ennemi que vous aurez à combattre est aussi brave et aussi habile que vous, bien armé et bien équipé. Mais vous allez venger la mort de notre représentant, celle de nos compatriotes mais aussi celle de nombreux autres Européens! Sus à l'ennemi, écrasez-le! Pas de pitié! Pas de prisonniers! Celui qui vous tombera sous la main est un homme mort : il y a mille ans, les Huns du roi Attila se sont fait un nom qui retentit formidablement aujourd'hui encore dans les mémoires et les contes; que le nom des Allemands acquière en Chine la même réputation, pour que jamais plus un Chinois n'ose même regarder un Allemand de travers ${ }^{54}$ !"

Léon Silbermann relativise plus loin ses propos : ainsi, estime-t-il que si les Allemands sont craints par les Chinois, c'est certainement le cas des soldats de toutes les nations, et donc des Français aussi : «Les Français étaient partout bien accueillis, tandis que les Allemands inspiraient la peur. Les Chinois disaient que les Fagoa (Français) sont bons et que les Tagoa (Allemands) sont méchants. Il est d'ailleurs probable qu'en présence des Allemands ils disaient le contraire ${ }^{55}$."

Ainsi, si toutes les nations collaborent sur le terrain et semblent animées par un réel désir de concorde, nos trois diaristes n'hésitent-ils pas à formuler quelques reproches à l'égard de la violence extrême des soldats allemands. On peut néanmoins se demander si cette bonne entente reste réelle jusqu’à la fin de la campagne.

\section{La coalition à l'épreuve du temps}

À la lecture du journal de Jules Bedeau on constate que les relations entre les alliés demeurent bonnes jusqu'à la fin de la campagne de Chine. Les soldats des nations coalisées échangent des marques de sympathie tout au long de l'expédition. Alors qu'il participe au défilé organisé en l'honneur du général Voyron, le 6 mars 1901, à Tien-Tsin, Jules Bedeau écrit : " Derrière lui et en côté, il y a plusieurs grandes tombes chinoises, elles sont couvertes de curieux, principalement des officiers étrangers parmi lesquels on remarque beaucoup d'Allemands et de Russes. Il y en a de toutes les autres nations mais en moins grand nombre ${ }^{56}$."

Ainsi, au printemps 1901, les alliés affichent-ils leur bonne entente. Le 29 juin, alors que notre artilleur et son unité quittent la Chine, des soldats d'une canonnière russe les " saluent avec enthousiasme ${ }^{57}$ ". De même, le 14 juillet est célébré dans le port de Hong-Kong par des navires anglais et américains ${ }^{58}$. Léon Silbermann observe la même scène alors qu'il est ce jour-là à Port-Saïd. Ainsi, l'harmonie règne-t-elle entre les nations jusqu'à la fin de l'expédition.

54. MiLzA, Pierre, Les Relations internationales de 1897 à 1914, Paris, 1968, p. 156.

55. SILBERMANn, Léon, Souvenirs..., op. cit., p. 224.

56. BEDEAU, Jules, Journal..., op. cit., p. 34.

57. Ibidem, p. 40.

58. Ibid., p. 47. 
À cette situation idéale entre les forces alliées, Jules Bedeau apporte un bémol à la date du 15 juillet 1901. Sa remarque concerne alors les Anglais. Alors qu'il longe, sur le chemin du retour vers la France, la côte méridionale de l'Empire du Milieu et qu'il observe un énorme dépôt de charbon, il écrit : " On dit que c'est le principal dépôt de charbon des côtes de Chine. Il appartient aux Anglais et serait une menace pour nos colonies. Mais les marins de la Vipère qui ont été longtemps ici, nous disent qu'en cas de guerre, nous nous en emparerions dès le début ${ }^{59}$. " Jules Bedeau évoque l'éventualité d'un conflit avec l'Angleterre. Pense-t-il à un affrontement direct ou plutôt à une querelle coloniale? Il n'apporte pas de précision. Le lecteur constate uniquement que pour Jules Bedeau et les marins de la Vipère, une guerre avec les Anglais est concevable. L'absence de justification au sujet de cet éventuel conflit à l'attention du lecteur peut faire penser que pour le lecteur français de 1900, un affrontement avec les forces anglaises est possible. Il est vrai que la guerre des Boxers se déroule deux années après les événements de Fachoda ${ }^{60}$; Anglais et Français sont alors en pleine rivalité pour le partage du monde. La coalition qui s'est formée à l'occasion de la guerre des Boxers apparaît fragile. Il ne s'agit donc que d'une alliance de circonstance. On retrouve chez Léon Silbermann cette défiance, voire cette hostilité, à l'égard des troupes anglaises. À de nombreuses reprises il critique l'attitude des soldats de l'armée anglaise ${ }^{61}$. Il affirme même : " Puis, les Anglais commencèrent à soulever quelques difficultés au fond desquelles on retrouvait toujours le souci de leur confort personnel. Leurs procédés à propos de tout leur valurent l'animosité des autres soldats ${ }^{62}$."

D'après Léon Silbermann, l'ensemble des soldats n'appréciait pas l'armée anglaise. Il évoque même à plusieurs reprises des accrochages entre des soldats britanniques et des soldats russes et allemands ${ }^{63}$. Une partie de la presse française témoigne aussi des tensions existant entre les Anglais et les autres alliés. Dès le 4 et le 8 août 1900, le journal La Sarthe s'inquiète de l'attitude des Britanniques et affirme que leur gouvernement ne se soucierait pas des intérêts communs et ne serait préoccupé que par l'extension de son autorité sur la vallée du Yang-Tsé et sur la région de Shanghaï. D'après le quotidien, l'ensemble des puissances serait alors en désaccord avec l'Angleterre.

La lecture des lettres du lieutenant britannique Bernard Holbrooke montre que ce sentiment d'animosité des Français à l'égard des Anglais est réciproque. Il critique en particulier la tenue des soldats français : ils sont

59. Ibid., p. 48.

60. En 1898, la France avait tenté de devancer l'Angleterre sur le haut Nil en y envoyant la mission Marchand qui, rejointe par Kitchener, dut évacuer Fachoda sur l'ordre de Delcassé après un ultimatum britannique. Cet échec, durement ressenti en France, entraîna l'accord franco-anglais de mars 1899 qui consacra le renoncement de la France à la région du nord du Nil.

61. SilBERMANN, Léon, Souvenirs..., op. cit., p. 201, 243 et 267.

62. Ibidem, p. 270 .

63. Ibid., p. 214, 243 et 248. 
sales et débraillés. Cela reflète leur amateurisme et leur manque de rigueur militaire. D'après Bernard Holbrooke, les soldats français sont méprisés par tous et cela est justifié ${ }^{64}$. Il est vrai qu'en 1900, les Britanniques ont le sentiment légitime d'appartenir à la plus grande puissance d'alors ${ }^{65}$. Cela explique peut-être la suffisance avec laquelle ces soldats semblent traiter les autres soldats de la coalition. Il est possible que leur position de force fasse naître chez les militaires des autres nations un sentiment de jalousie. Le fait que les soldats anglais sont alors les mieux rémunérés et mènent par conséquent un train de vie supérieur aux autres ajoute peut-être encore à la jalousie. Ainsi, seuls les soldats britanniques sont-ils l'objet de reproches systématiques.

Les Russes ne sont pas pour autant épargnés par les critiques. Leur extrême violence à l'égard des Chinois est dénoncée par le soldat Silbermann ${ }^{66}$. Georges Weulersse, présent en Mandchourie au mois de juillet 1900 , souligne aussi leur violence. Il est difficile de donner une explication à cette attitude. Léon Silbermann constate seulement que les soldats du Tsar tirent facilement. Léon Silbermann indique que les soldats français en font la cruelle expérience. Il rapporte en effet qu'un détachement de zouaves a été la cible, le 2 octobre 1900, des tirs des soldats russes. N'ayant pas identifié l'uniforme particulier des zouaves qu'ils confondent avec l'habit habituel des Boxers, les Russes ouvrent le feu sur les soldats français, sans sommation. Deux hommes sont tués et sept blessés. Aux yeux de Léon Silbermann cette attitude caractérise bien le comportement irréfléchi des Russes durant le conflit. L'événement et la sépulture avec les honneurs militaires des victimes sont largement évoqués dans le journal L'illustration $^{67}$. Le soldat Silbermann nous apprend, dans un lyrisme teinté d'exagération, que les Russes, bien que violents, furent terriblement affectés par leur méprise : "Quand l'erreur fut reconnue, les Russes vinrent embrasser les zouaves en pleurant; les larmes coulaient, mais l'irréparable était fait ${ }^{68}$."

Ainsi, si les relations entre les soldats des nations coalisées décrites par nos trois diaristes sont bonnes, il apparaît qu'à mesure que la campagne se prolonge des critiques naissent à l'endroit des Russes et des Anglais. L'animosité exprimée à l'égard de ces derniers semble beaucoup plus profonde. À la lecture de Léon Silbermann, on constate néanmoins qu'avec le temps, le principe même de la coalition semble perdre son sens ${ }^{69}$ : "Après la prise de Pékin, le système des alliés fut du dernier ridicule. [...] Il est permis de supposer que les puissances avaient envoyé des troupes en

64. HolBROOKE, Bernard Frederick Roper, China, 1900 : The Eyewitnesses Speak, Londres, Green-Hill Books, 2000, p. 237.

65. KeIGER, John, «La perception de la puissance française par le Foreign Office ", $L a$ puissance française à la belle époque, p 175 à 199.

66. Ibid., p. 268.

67. VILLETARD DE LAGUÉRIE, L'Illustration, n 3020, 12 janvier 1901, p. 23.

68. SilBermann, Léon, Souvenirs..., op. cit., p. 211.

69. Ibidem, p. 214 et 228. 
Chine, non pour accomplir une œuvre humanitaire mais pour se partager éventuellement le territoire. Chacune cherchait naturellement le plus gros morceau $^{70}$."

Léon Silbermann aurait pu ajouter que c'est surtout la répartition entre les nations coalisées de la somme que devrait verser la Chine aux puissances à l'issue du conflit, qui constituait la principale source de tensions entre elles. Si Léon Silbermann évoque une dégradation des relations entre les gouvernements des différentes puissances en Chine, en revanche il ne constate pas de détérioration de celles entre les soldats sur le terrain.

On peut en particulier souligner que même si la coalition perd de son sens avec le temps, les soldats japonais ne sont en revanche, à aucun moment, l'objet de critiques de nos trois diaristes. Ils jouissent d'une image très positive en particulier dans le journal de Léon Silbermann, qui nous apporte l'essentiel de nos informations à leur sujet. Ils lui apparaissent comme des alliés à part entière et le Japon est regardé comme une nation appartenant à la grande famille des nations dites civilisées. Léon Silbermann rédige un long développement, teinté d'admiration, sur l'organisation de l'armée japonaise qui a été structurée par des officiers européens : «L'armée japonaise a été instruite en premier lieu par des Français, à compter de 1871. En outre, en 1879, le Japon a fait appel dans le même but à des officiers allemands. Ceux qui ont vu l'armée japonaise à l'œuvre s'accordent à dire qu'elle peut parfaitement être comparée avec les troupes européennes ${ }^{71}$."

Les soldats français et japonais jouent un rôle décisif lors de la prise de Tien-Tsin. Les militaires français ont alors l'occasion de constater les qualités de leurs alliés ${ }^{72}$. Etablissant un tableau des différentes armées engagées contre la Chine, Léon Silbermann loue les qualités exceptionnelles des soldats japonais : " Les plus petits [soldats] étaient les Japonais; mais ces derniers à défaut de taille, avaient d'excellentes qualités militaires. Ils étaient très disciplinés, adroits tireurs et semblaient dévoués à leurs chefs jusqu'à la mort. Leurs armes et leur matériel de transport étaient d'une propreté remarquable ${ }^{73}$."

Les autres diaristes adoptent le même type de position quant aux soldats japonais, mais ils les évoquent de façon plus laconique. C'est parce que le Japon s'ouvre à l'Occident et qu'il accueille positivement la modernité depuis l'ère Meiji que nos trois soldats le perçoivent de façon si positive. Pierre Foucault a insisté, dans un article sur l'image de l'Asie dans la presse sarthoise entre 1841 et 1914, sur l'image positive qui y était donnée du Japon ${ }^{74}$. Les Japonais y apparaissent comme rigoureux, disciplinés et

70. Ibid., p. 251.

71. Ibid., p. 193

72. Ibid., p. 195.

73. Ibid., p. 193.

74. FouCAulD, Pierre, " La presse sarthoise et l'Asie ", Annales de Bretagne et des Pays de l'Ouest, Tome 112, 2005, nº 3, p. 142 à 173. 
travailleurs. On peut imaginer que le contenu des quotidiens dans le reste de la France était assez proche des quotidiens sarthois. Il n'apparaît donc pas surprenant que nos trois diaristes portent de tels jugements à l'égard des Japonais. En escale à Nagasaki, lors du voyage retour vers la France, Jules Bedeau constate la modernité du Japon qui contraste avec le faible développement économique de la Chine. Il observe aussi l'importance de la présence étrangère, témoignage de l'ouverture du Japon à l'Occident. Le Japon appartient bien alors au concert des nations civilisées et ses soldats sont dignes de respect ${ }^{75}$.

Ainsi, la lecture des diaristes français de la guerre des Boxers laisse-t-elle entrevoir l'existence d'une réelle entente entre la plupart des soldats des armées des nations occidentales et japonaise. On retiendra, en particulier, la très bonne qualité des relations entre les militaires français et allemands. Il y a bien sûr quelques tensions, quelques accrochages sur le terrain, mais globalement la nécessité de se rapprocher, pour lutter contre un ennemi commun, l'emporte sur les autres considérations. Apparaissent alors les limites des sources sur lesquelles s'appuient nos propos. En effet, on ne peut savoir quelles sont les intentions plus lointaines des états-majors et de leurs gouvernements. En 1900, les rivalités restent importantes entre la plupart des gouvernements des nations coalisées, en quelque sorte, malgré elles. Néanmoins, la lecture des journaux des diaristes de la guerre des Boxers permet de constater avec quelle facilité des soldats, dont les armées ont été parfois opposées quelques années auparavant et qui s'affronteront quatorze années plus tard, lors de la Grande Guerre, parviennent à coopérer et semblent habités par le désir de s'épauler. L'animosité qui caractérise les relations entre soldats anglais et français retient aussi l'attention. On mesure à quel point l'Entente Cordiale signée, quatre années plus tard, entre l'Angleterre et la France, constituera pour un grand nombre des habitants de ces deux pays un événement contre-nature. Ainsi, les sources utilisées pour rédiger ce document permettent-elles de s'interroger sur l'écart entre les positions diplomatiques des différents gouvernements et le ressenti des citoyens de ces mêmes pays.

75. BEDEAU, Jules, Journal..., op. cit., p. 42. 


\section{RÉSUMÉ}

Le soulèvement des Boxers éclate dans le nord de la Chine en juin 1900. Les Boxers, animés par un fort sentiment xénophobe, assiègent les étrangers dans les quartiers qui leur sont réservés : les légations. Inquiets pour leurs ressortissants, les gouvernements russe, britannique, américain, allemand, autrichien, italien, français et japonais organisent l'envoi d'un corps expéditionnaire international afin de libérer les assiégés. Les soldats des huit nations parviennent le 14 juillet à libérer la légation de Tien-Tsin et le 15 août celle de Pékin. Parmi le contingent français, certains soldats, conscients qu'ils vivent un moment unique à l'échelle de leur vie dans ce pays lointain, prennent la plume et s'engagent dans l'écriture de journaux de campagne. Ces écrits constituent une source passionnante pour appréhender l'image des soldats étrangers à travers le regard de simples hommes de troupe. Si l'entente entre les soldats des huit nations constitue au départ une nécessité vitale face au danger que constituent les Boxers, il semble que la concorde entre les militaires français et allemands se poursuit durant toute la campagne. Le regard porté sur les soldats japonais apparaît aussi éminemment positif. A l'opposé, les relations avec les soldats britanniques se dégradent rapidement. Ceux-ci font l'objet de nombreuses critiques. Quant aux Russes, ils sont critiqués pour leur violence extrême à l'égard des populations chinoises.

\section{ABSTRACT}

The uprising of the Boxers broke out in Northern China in June 1900. Strongly imbued with xenophobia, the Boxers besieged the strangers in their reserved quarters: the legations. Worried by their nationals, the Russian, British, American, German, Austrian, Italian, French and Japanese governments deployed international expeditionary forces in order to free the besieged. The eight nations' soldiers managed to liberate the Tien-Tsin legation on July $14^{\text {th }}$ and Peking's on August $15^{\text {th }}$. Among the French contingent some soldiers started to write out campaign diaries, fully realizing they were experiencing once-in-a-life-time moments in this distant country. The pieces of writing make up a fascinating source to picture the foreign soldiers through the eyes of the privates. Whereas the harmony of the eight nations' soldiers was an absolute necessity to face the Boxers' danger, the concord of the French and German military seemed to go on throughout the campaign. The Japanese soldiers also appeared to be regarded very highly. On the contrary, the relations with British soldiers quickly deteriorated. They were subjected to a lot of criticism. As for the Russian, they were criticized for their extreme violence toward the Chinese populations. 